\title{
La prothèse complète immédiate d'usage : difficultés et gestion
}

\section{The immediate complete prosthesis of custom: difficulties and management}

\section{Résumé}

\section{MOTS-CLEFS :}

- Prothèse immédiate, résorption osseuse, maintenance

\section{KEYWORDS:}

- Immediate prostesis, bone resorption, maintenance

$\operatorname{AOS} n^{\circ} 289-2018$
$\mathrm{Vu}$ les multiples avantages qu'offre la réhabilitation prothétique par une prothèse complète immédiate d'usage, elle demeure toujours une solution de choix devant l'urgence esthétique. Cependant cet acte prothétique reste très délicat à perfectionner puisqu'il expose plusieurs difficultés à gérer durant toutes les étapes cliniques. La présence des dents mal positionnées, toujours très mobiles constitue le principal problème qui complique toutes les étapes prothétiques et à qui tous les autres problèmes sont reliés.

\section{Abstract}

\author{
Given the multiple benefits of prosthetic \\ rehabilitation with a full prosthesis \\ immediate use, it remains a solution \\ of choice to aesthetics emergency. \\ However, this prosthetic act remains \\ very difficult to perfect as it presents \\ several difficulties in managing all \\ clinical stages. The presence of poorly \\ positioned teeth, always moving is \\ the main problem that complicates \\ all prosthetic steps and that all other \\ problems are connected.
}

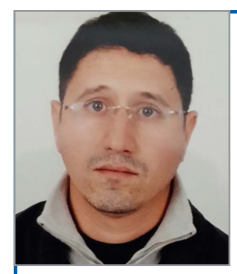

- Mohssine BAGUI, Professeur assistant en prothèse adjointe à la faculté de médecine dentaire de Rabat.

Leila FAJRI, Professeur agrégé en prothèse adjointe à la faculté de médecine dentaire de Rabat.

Khalifa BELHAJ, Résidant en odontologie chirurgicale CCTD, Rabat.

Bouabid EL MOHTARIME, Professeur de l'enseignement supérieur en prothèse adjointe. Chef de service d'odontologie à l'hôpital d'instruction militaire Mohammed V, Rabat.

Nadia MERZOUK, Professeur de l'enseignement supérieur en prothèse adjointe. Chef de service de prothèse adjointe CCTD, Rabat.

L a perte brutale des dernières dents, surtout antérieures, présente toujours un choc psychologique pour chaque patient candidat à l'édentation totale vu leur valeur psychique et les séquelles esthétiques et fonctionnelles de cet acte irréversible. Les patients n'acceptent en aucun cas de montrer cet handicap même pour une courte durée $[1,2]$.

Actuellement, l'implantologie offre plusieurs solutions pour la réhabilitation immédiate de ce type d'édentation, mais devant des contre-indications à l'implantation, la prothèse complète immédiate d'usage s'impose comme solution d'un grand intérêt.
Cette réhabilitation, comme son nom l'indique, n'est ni transitoire ni provisoire. Il s'agit de l'élaboration d'une prothèse d'usage posée le jour même de l'extraction des dernières dents.

La prothèse complète immédiate d'usage présente plusieurs avantages justifiant son intérêt. La mise en place de la prothèse d'usage, la même séance que les extractions, permet une meilleure organisation du caillot sanguin, une minimisation d'œdème, une restauration des fonctions de l'appareil mandicateur et un confort postopératoire considérable pour le patient. D'autant plus que cette prothèse guide la cicatrisation, et assure un soutien immédiat des organes para prothétiques. L'avantage 
majeur de cette éventualité thérapeutique réside dans la minimisation de la résorption osseuse à court et à moyen terme, avantage inexistant, lors de la réhabilitation par prothèse provisoire ou transitoire $[3,4]$. Cependant, ce type de réhabilitation présente plusieurs difficultés à gérer durant toutes les étapes de réalisation, qui doivent être menées avec beaucoup de rigueur. Avant tout, le clinicien doit pratiquer un examen clinique complet, poser la bonne indication, organiser un plan de traitement bien détaillé et préparer le patient convenablement avant d'entamer la réhabilitation prothétique proprement dite. Pour un meilleur résultat, le praticien doit prendre des empreintes statiques et hémodynamiques précises, déterminer, enregistrer et transférer un rapport intermaxillaire correct, faire un choix des dents prothétiques respectant les documents pré-extractionels, réaliser une rectification du modèle de travail en prévoyant la résorption et en corrigeant les contre dépouilles, assurer un montage esthétique correct sans avoir la possibilité d'effectuer un essayage en bouche, établir une occlusion statique et dynamique parfaitement stable, mener un acte chirurgical guidé très précis précédé par des extractions atraumatiques et enfin instaurer un suivi régulier pour maintenir un résultat satisfaisant [5].

\section{LA PRÉPARATION DU TERRAIN}

Un abord psychologique rationnel et bien mené, instaure un climat de confiance et une relation médecin malade facilitant la prise en charge et poussant le patient à coopérer durant toutes les séances cliniques. La motivation à l'hygiène bucco-dentaire, suivie d'un détartrage, diminue la charge bactérienne en bouche permettant ainsi de mener les étapes prothétiques dans un environnement assaini, ce qui permet une bonne cicatrisation.

Afin de faciliter les étapes de réalisation et optimiser le résultat final, il est recommandé d'extraire les dents postérieures et mettre ainsi le patient dans une situation d'édentement classe I Kennedy Applegate (3).

\section{LES EMPREINTES}

Pour ce type de prothèse, l'empreinte doit répondre aux critères requis pour l'empreinte en prothèse amovible totale, malgré la présence des dents sur l'arcade. Prendre des empreintes précises sans avulsion des dents généralement très mobiles en malposition, et en présence de diastèmes, reste un acte à haut risque.

Fig. 1 :

Porte empreinte individuel fenestré.
Assurer un joint périphérique antérieur étanche et hermétique en présence de contre dépouille antérieure et des dents souvent vestibulo versées, rend illusoire la désinsertion du porte empreinte après prise du matériau. Lors de l'enregistrement du comportement hémodynamique de la fibromuqueuse le praticien doit prendre en considération le degré de mobilité des dents et la différence de dépressibilité entre la fibromuqueuse et le desmodonte $[3,4,5,6]$.

\section{Empreinte primaire muco statique}

Avant de prendre l'empreinte à alginate, il est recommandé de combler les diastèmes inter-dentaires par un silicone lourd afin d'éviter d'extraire les dents lors de la désinsertion. Afin d'éviter tout manque en présence de contre dépouille exagérée, il est préférable de mettre directement en bouche de l'alginate sur le site concerné avant d'insérer le porte empreinte garni. Sur le plan pratique, il est souhaitable d'utiliser de l'eau glacée dans le but d'allonger le temps de prise ce qui offre au praticien suffisamment de temps pour déposer le matériau dans les zones où le risque d'avoir un manque est considérable.

On peut même envelopper les dents très mobiles dans une clé de matériau élastique avant de la surmonter par une empreinte globale à alginate et ceci afin d'éviter l'avulsion des dents lors de la désinsertion du porte empreinte en profitant de la grande élasticité du matériau utilisé (3), (4), (5), (6).

\section{Porte empreinte individuel}

Le porte empreinte individuel doit être bien adapté au niveau de la fibromuqueuse pour enregistrer le degré de dépressibilité, espacé et fenestré au niveau des dents afin de réduire l'encombrement antérieur et permettre un enregistrement du joint antérieur le plus physiologique possible Les bords doivent être à $2 \mathrm{~mm}$ du fond du vestibule même au niveau des dents. De plus la fenestration minimise le risque de fracture des dents en plâtre, lors du démoulage de l'empreinte $[3,4,5,6,7]$ (Fig. 1).

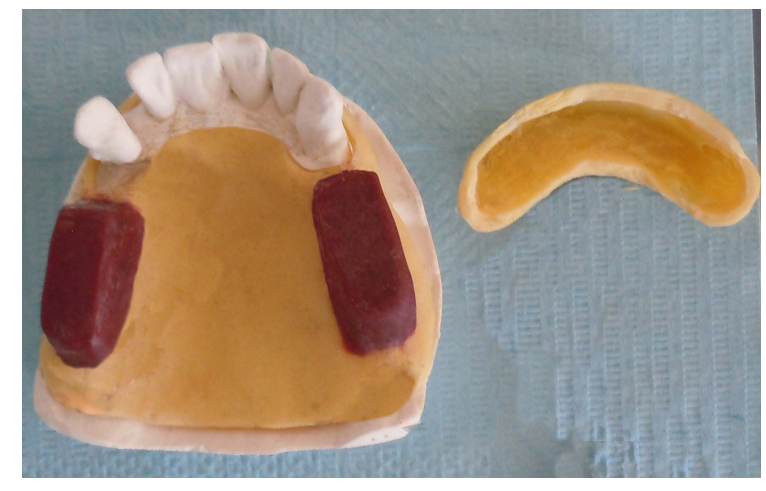




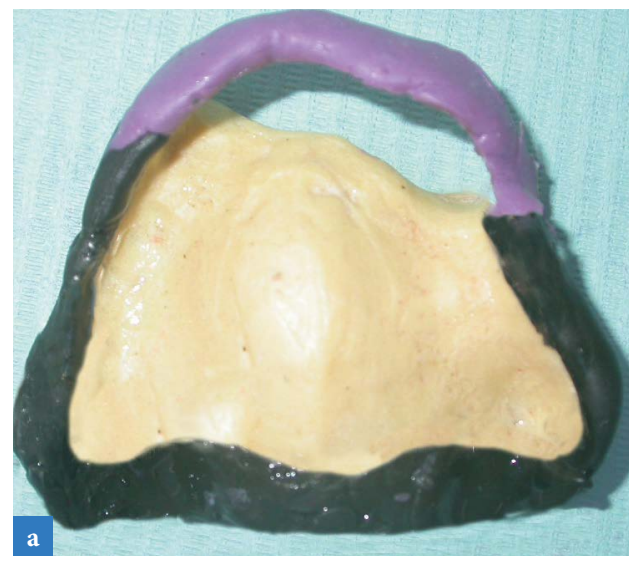

$\triangle$ Fig. 2-a : Remarginage avec un matériau plastique au niveau antérieur.

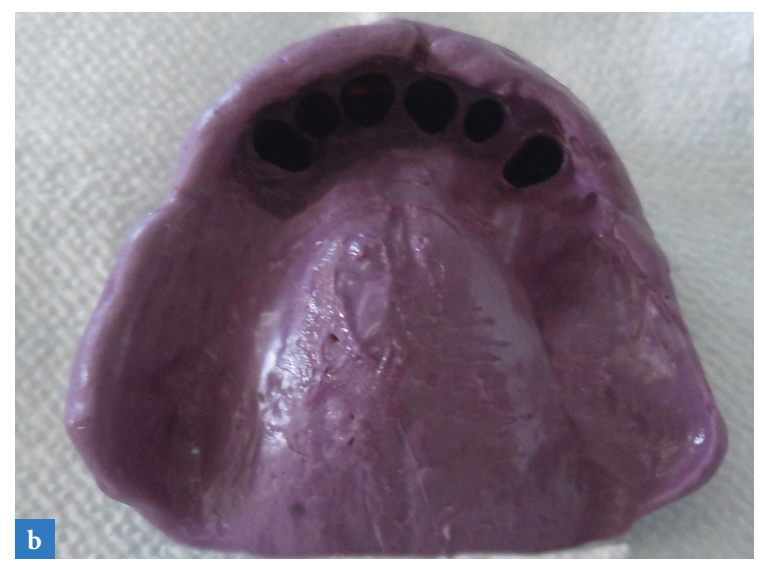

$\triangle$ Fig. 2-b : Empreinte secondaire en utilisant un polyéther.

\section{Empreinte secondaire anatomo-fonctionnelle}

Le remarginage au niveau postérieur peut se faire par une pâte thermoplastique, en revanche le joint antérieur doit être souple afin de dépasser aisément la contre-dépouille antérieure lors de la désinsertion. Les polyéthers de moyenne viscosité type "permadyne» sont les plus indiqués devant une telle situation (Fig. 2-a).

Le surfaçage au niveau édenté peut-être fait en utilisant la pâte à oxyde de zinc, par contre au niveau denté l'utilisation d'un matériau élastique est de règle. Pour plus de précisions, il est recommandé d'appliquer un matériau light soutenu par une moyenne viscosité. Les polysulfures type "permlastic", matériaux très élastiques trouvent toute leur indication dans des conditions pareilles (Fig. 2-b).

Le comblement des espaces inter-dentaires avant la prise d'empreinte élimine tout risque d'avulsion dentaire $[3,4,5,6]$.

\section{LE RAPPORT INTER MAXILLAIRE}

La présence des dents constitue un élément perturbateur pour la détermination et l'enregistrement du rapport maxillo mandibulaire vu les malpositions, les égressions et les mobilités. Lors de l'enregistrement du rapport, tout contact dento-dentaire doit être considéré comme contact perturbateur.

Les maquettes d'occlusion doivent être parfaitement stables en bouche et sur modèles.

Les points inter incisifs supérieur et inférieur doivent être matérialisés en premier selon l'axe sagittal médian. Le rapport inter maxillaire doit être déterminé sans contact entre les dents ni entre les dents et les bourrelets (Fig. 3-a, b, c).

Le plan d'occlusion doit être parallèle à la ligne bi pupillaire et au plan de Camper en prenant en considération les égressions dentaires. On peut légèrement l'abaisser par rapport à son niveau idéal afin d'éviter les contacts perturbateurs déjà cités.

La DVO est réglée selon les critères esthétiques, phonétiques et de déglutition comme pour la prothèse totale conventionnelle. On est fréquemment obligé d'enregistrer le rapport selon une dimension verticale d'enregistrement légèrement surestimée par rapport à la dimension verticale d'occlusion souhaitable dans le but d'éviter tout contact des dents entre elles, ou avec les bourrelets.

Avant la mise en articulateur, il faut ajouter à la tige incisive la valeur mesurée (différence entre DVE et DVO souhaitable). Et après transfert sur articulateur, elle sera repositionnée à 0 .
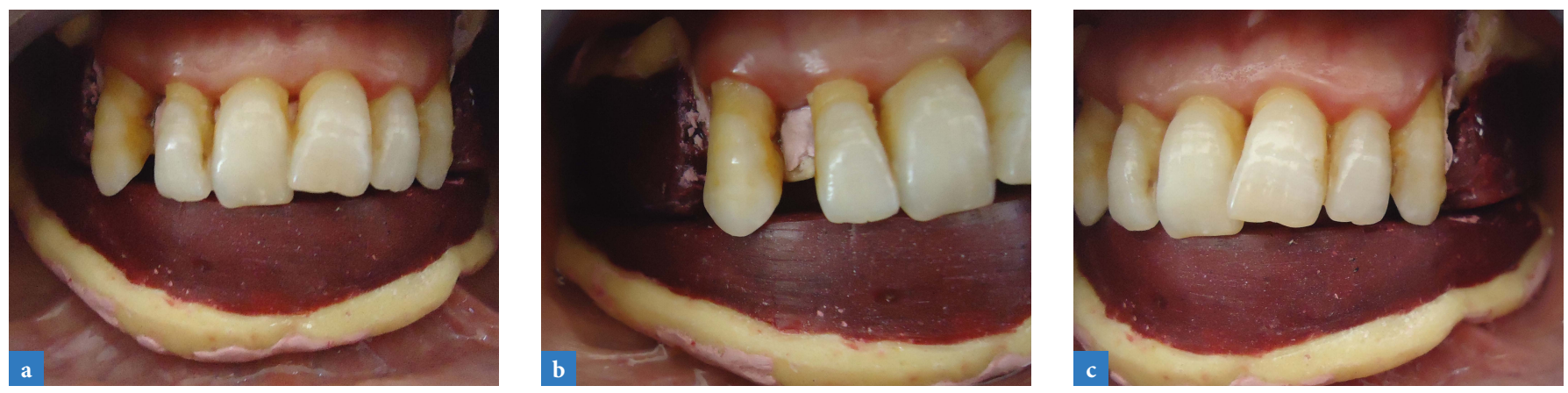

$\triangle$ Fig. 3-a, b, c : Rapport inter-arcade avec contact bourrelet-bourrelet. 
Le rapport inter maxillaire est toujours enregistré à la position de relation centrée (Fig. 4). Il ne faut jamais enregistrer ce rapport selon l'occlusion du patient puisqu'elle est la résultante de plusieurs perturbations et pathologies occlusales. L'utilisation d'arc facial pour le transfert du rapport sur l'articulateur est toujours de règle afin de pouvoir passer de DVE à la DVO souhaitée sans fausser le rapport [3, 8, 9, 10] (Fig. 5).

\section{CHOIX ET MONTAGE DES DENTS PROTHÉTIQUES}

Le choix des dents prothétiques sera guidé par les dents résiduelles, en forme, en teinte et en dimension (Fig. 6). Les dents postérieures seront montées selon

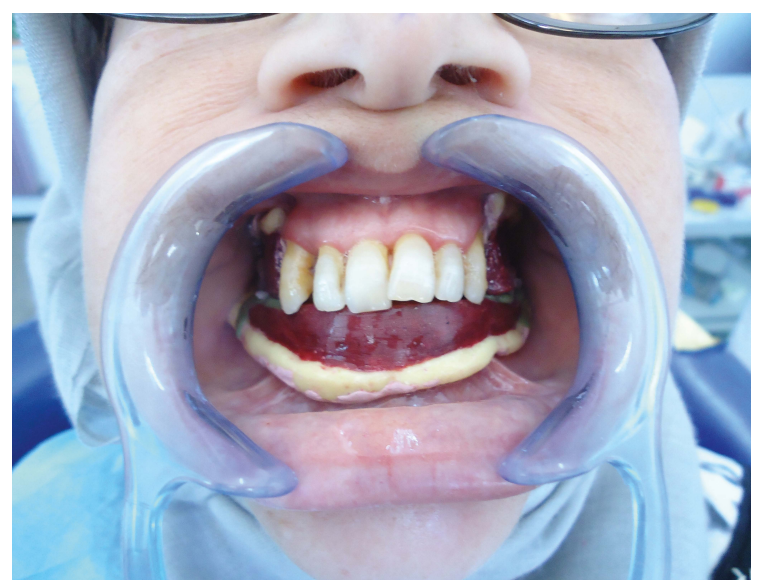

$\triangle$ Fig. 4 : Enregistrement du rapport inter-maxillaire en position centrée.

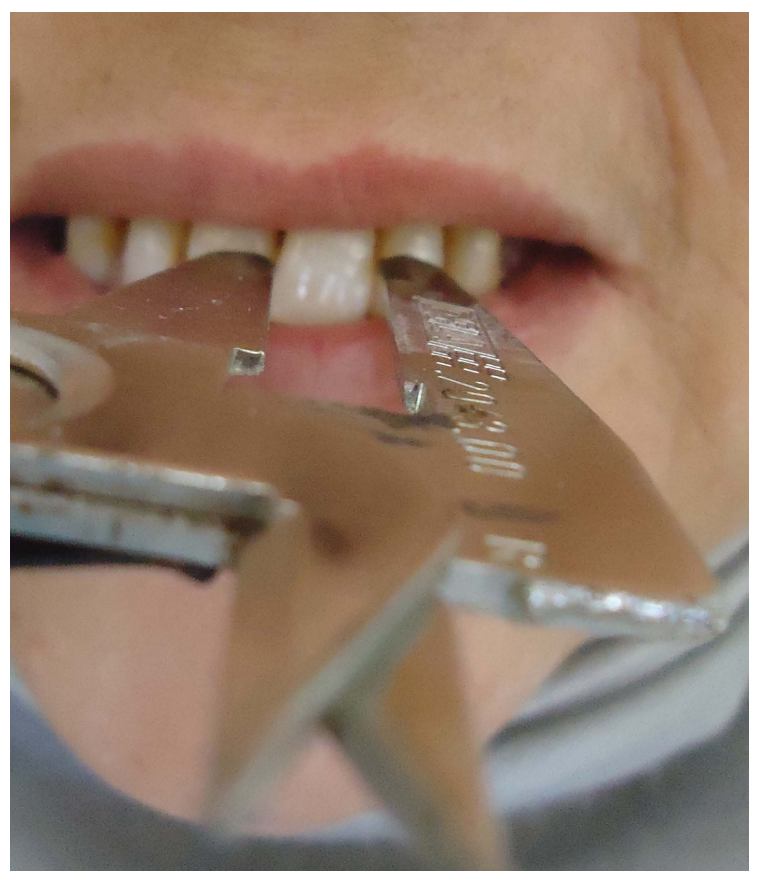

$\triangle$ Fig. 6: Utilisation des documents pré-extractionnels afin d'orienter le choix des dents prothétiques. le rapport déjà enregistré en respectant le concept de l'occlusion intégralement équilibrée.

Un essayage fonctionnel permettra de confirmer en partie le choix des dents, mais surtout la fiabilité et la précision du rapport enregistré.

Avant la rectification du modèle secondaire qui doit être dupliqué, il est recommandé de réaliser une clé, en silicone lourd, mordue et une autre vestibulaire qui seront utilisées comme référence afin de positionner les dents antérieures prothétiques dans une position correcte, dans l'aire de sustentation, selon le point inter incisif préalablement déterminé et selon les critères esthétiques (Fig. 7).

Le respect du concept de l'occlusion intégralement équilibrée est une nécessité indiscutable pour l'équilibre occlusal de la future prothèse $[3,5,10,11]$.

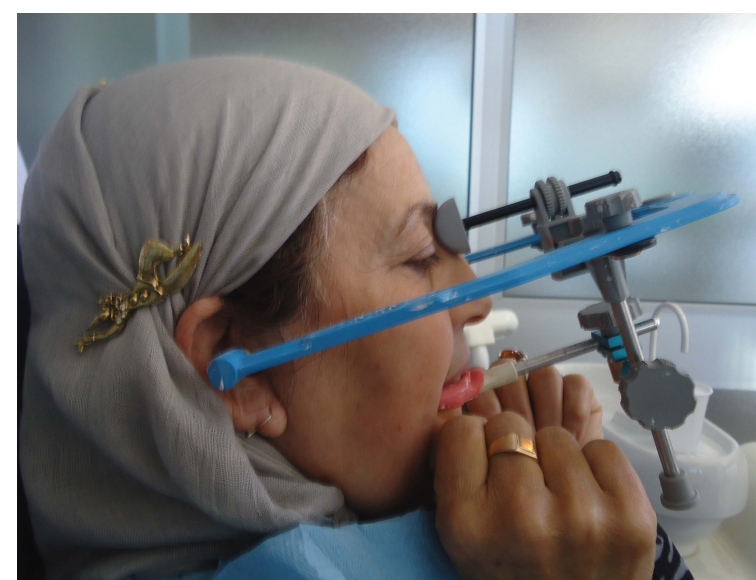

$\triangle$ Fig. 5 : Transfert du rapport enregistré à l'aide d'un arc facial.

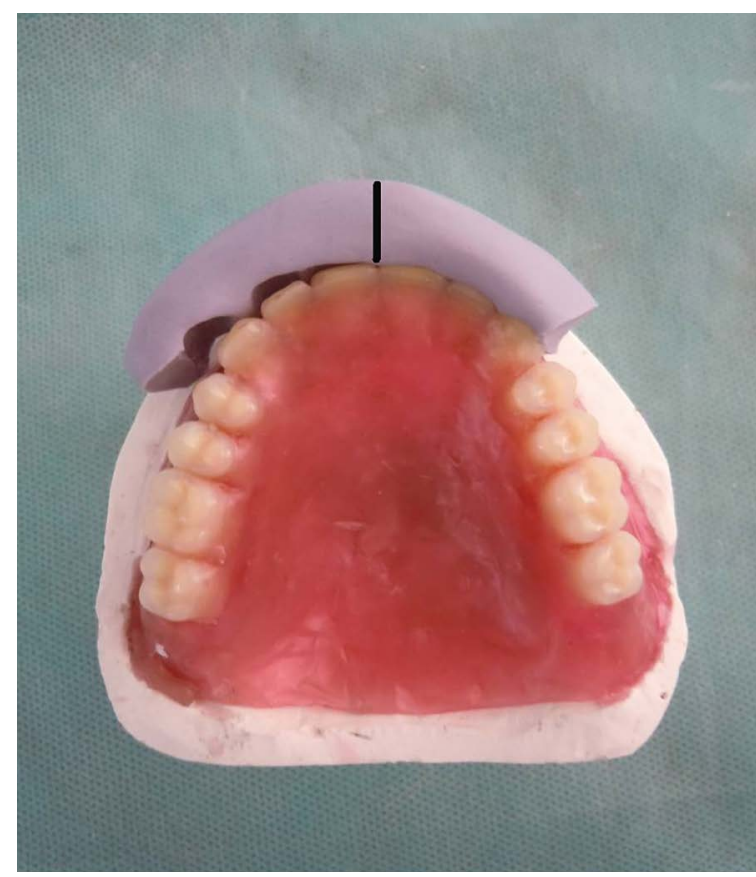

$\triangle$ Fig. 7 : Clé siliconée matérialisant les malpositions et orientant les corrections lors du montage. 


\section{RECTIFICATION DU MODĖLE SECONDAIRE}

Cette étape consiste à l'élimination des dents résiduelles en plâtre et la correction de la contre-dépouille en prévoyant l'espace nécessaire pour la fausse gencive et les dents prothétiques afin d'assurer un soutien labial optimal et une insertion-désinsertion facile de la future prothèse.

Ces rectifications seront guidées par les clés en silicone déjà citées, par le bilan radiologique ainsi que par les valeurs du sondage parodontal. Le degré de lyses osseuses et des récessions est en rapport significatif avec le degré de résorption que présentera le site après extraction et régularisation osseuse. Il est strictement interdit de toucher le fond du vestibule puisqu'il n'est pas concerné par la résorption et cet acte ne causera que l'altération de la qualité de la rétention prothétique $[3,5,10,11]$.

\section{L'ÉTAPE CHIRURGICALE}

Un guide chirurgical, copie conforme de la future prothèse, devra être élaboré à partir du duplicata du modèle rectifié. Une fois en bouche et sous pression occlusale de préférence, ce guide en résine transparente, permettra de visualiser et matérialiser les zones de compression (Fig. 8).

Après avulsion des dents restantes, une régularisation osseuse guidée et sous pression occlusale sera réalisée pas à pas jusqu'à ce qu'elle assure une insertion désinsertion aisées et une absence totale de toute zone de compression. Il faut préserver au maximum la table osseuse externe et régulariser les septums inter dentaires $[3,5,10,11]$ (Fig. 9, 10).

\section{L'INSERTION PROTHÉTIQUE}

Pour minimiser l'œdème et pour que la prothèse épouse la surface d'appui, le patient sera amené à serrer sur deux rouleaux de coton durant dix minutes. De plus ceci permettra au patient de fermer en relation centrée. L'occlusion statique sera ensuite vérifiée pour obtenir des contacts occlusaux uniformément répartis [3, 5] (Fig. 11).

\section{MAINTENANCE}

Il sera strictement interdit au patient de retirer sa prothèse durant les premières 48 heures. Un premier nettoyage est recommandé après cette période, afin de

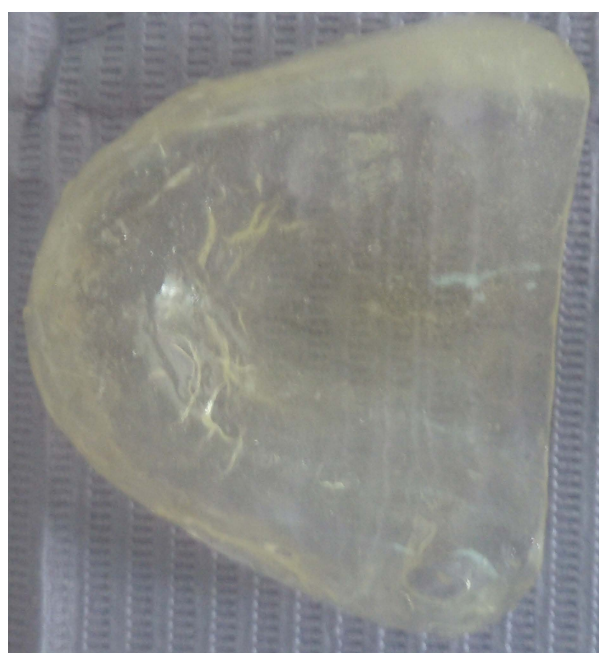

$\triangle$ Fig. 8 : Guide chirurgical issu d'un duplicata du modèle secondaire déjà rectifié.

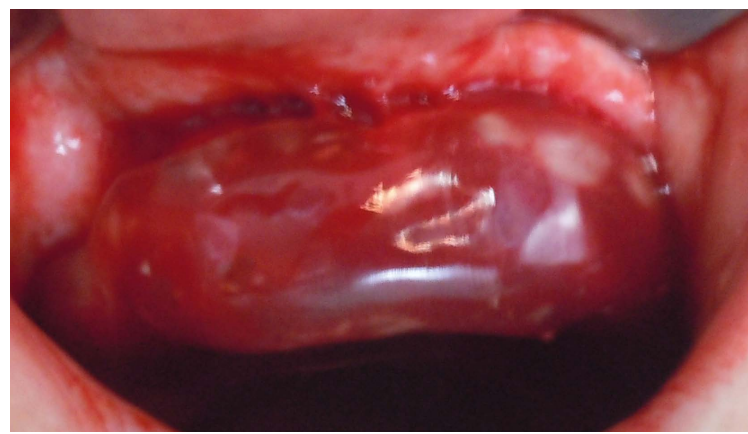

$\triangle$ Fig. 9 : Régularisation osseuse guidée.

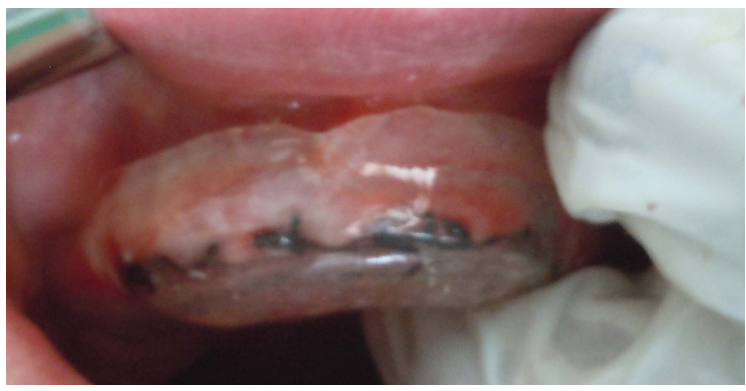

$\triangle$ Fig. 10 : Vérification de l’adaptation du guide après sutures.

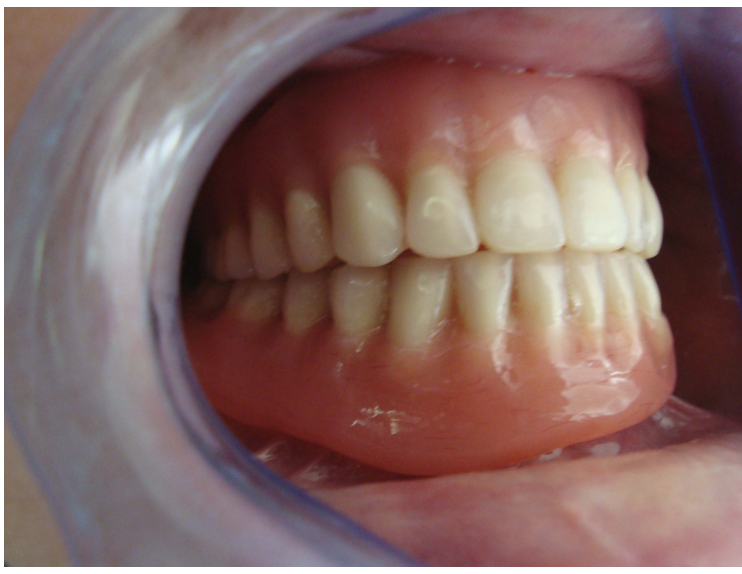

$\triangle$ Fig. 11 : Insertion prothétique et équilibration statique. 
vérifier la cicatrisation et affiner l'occlusion statique. Il est préférable de reporter la rectification de l'occlusion dynamique après disparition complète des suites opératoires (Fig. 12, 13).

Le premier mois les visites de contrôle doivent être faites chaque semaine, après seront programmées des visites à 3 mois, 6 mois, et un an $[3,5,10]$.

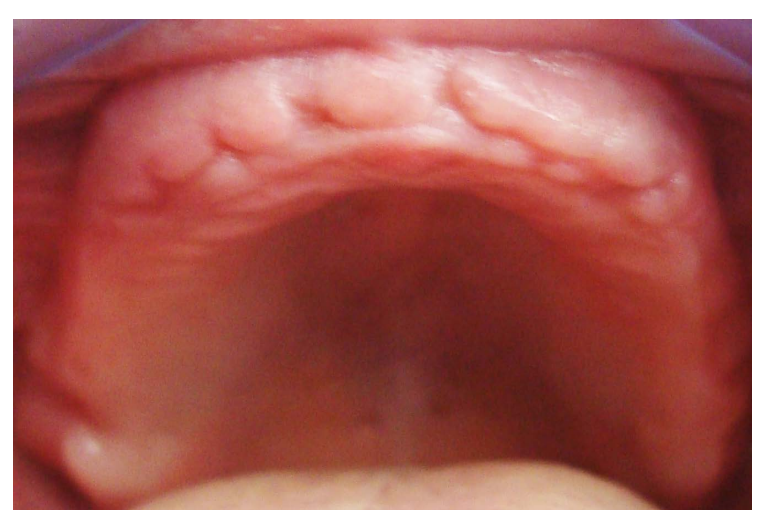

$\triangle$ Fig. 12 : Résultat après un mois de cicatrisation.

Fig. 13 : Résultat final et sourire de satisfaction.

\section{CONCLUSION}

Même-si la prothèse complète immédiate d'usage constitue toujours un défi pour le praticien. Elle reste un recours fréquemment rencontré en pratique quotidienne de la prothèse adjointe. Vu ses avantages, ce type de réhabilitation restera toujours d'actualité et gardera une place très importante dans notre discipline d'où l'intérêt de maitriser toutes ses étapes de réalisation cliniques et de laboratoires.

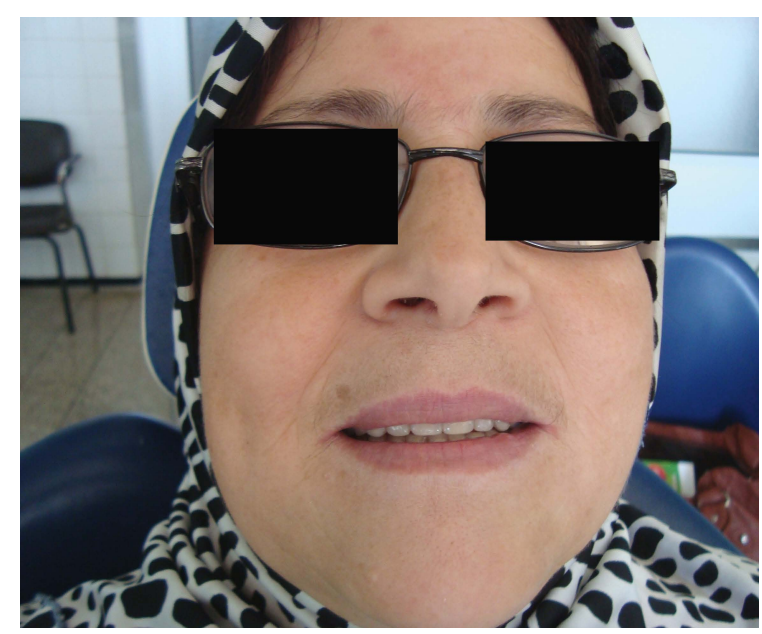

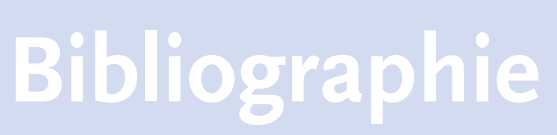

[1] Phoenix RD, Fleigel JD. Cast modification for immédiate complete dentures: traditional and contemporary considerations with and introduction of spatial modeling. J Prosthet Dent 2008 ; 100: 399-405.

[2] Smith RA. Immediate complete dentures; a starting point. J Am Dent Assoc 1973; 87 : 641-645.

[3] Hue O, Berteretche MV. Prothèse complète : réalité clinique, solutions thérapeutiques. Quint International édit, Paris, 2004.
[4] Herbout B, Postaire M. Prothèse complète immédiate d'usage ; Actualisation de la technique. Cah Proth 2000 ; 111: 55-65.

[5] Abdelkoui A, Fajri L, Benama A, Abdedine A. La prothèse complète immédiate d'usage : réalisation temps par temps. Clinic 2003 ; 34: 87-96.

[6] Rignon-Bret JM. Les empreintes en prothèse complète immédiate. Inf Dent 1988 ; 16: 1315-23.

[7] Dupuis V, Rimpaut L, Dabadie M, Galvin D. Intérêt des PEI fractionnés en prothèse immédiate. Inf Dent $1993 ; 30$ : 2157-63.
[8] Rignon-Bret JM. La détermination du rapport intermaxillaire en prothèse complète immédiate (1 ${ }^{\text {re }}$ partie). Inf Dent 1989 ; 31: 2703 14.

[9] Rignon-Bret JM. La détermination du rapport intermaxillaire en prothèse complète immédiate ( ${ }^{\mathrm{e}}$ partie). Inf Dent 1989 ; 36: 3367 78.

[10] Schoendorff R, Jeannin C. Prothèse immédiate. Encycl Med Chir, Odontologie, 23325-K-10, 1998.

[11] Berteretche $M$, Hue $O$. La prothèse complète immédiate, une entité clinique, mais différentes approches. Cah Proth 1998 ; 104: 89-101. 